\section{MS1-P8 EMBL P13 beamline and derivative laboratory at PETRA III \\ @DESY: phasing of biological macromolecules with softer X-rays and heavy atom derivatives}

Michele Cianci ${ }^{1}$, Gleb Bourenkov ${ }^{1}$, Johanna Kallio ${ }^{1}$, Gulliaume Pompidor $^{1}$, Stefan Fiedler ${ }^{1}$, Thomas Schneider ${ }^{1}$

\section{EMBL, Hamburg, Germany}

email: michele.cianci@embl-hamburg.de

For many heavy atom compounds the anomalous signal is enhanced when collecting data at low energies $(>2 \AA$ wavelength) compared to standard data collections at $1 \AA$ wavelength. The macromolecular crystallography beamline P13 is in operation since late 2012 and it is part of the European Molecular Biology Laboratory Integrated Facility for Structural Biology at PETRA III (DESY, Hamburg, Germany). P13 is tunable across the energy range from 4 to $17.5 \mathrm{keV}$ to support crystallographic data acquisition exploiting a wide range of elemental absorption edges for experimental phase determination. Data collections at energies as low as $4 \mathrm{keV}(3.1 \mathrm{~A})$ are possible due the optimized beam line design, the high photon flux of up to $2 \times 10^{10} \mathrm{ph} / \mathrm{sec}$ at $4 \mathrm{keV}$ ), the custom calibration applied to the PILATUS 6MF detector, and the availability of a partial Helium-path. This set-up allows exploiting very long wavelengths to harness the anomalous signal from heavy atoms for phase determination. Using adaptive X-ray mirrors and a set of apertures the beam size can be easily adjusted to the crystal size. The beam line offers rapid access to a heavy atom derivative laboratory equipped for solution preparation, soaking and crystal manipulation. The heavy atom library includes more than 150 compounds from more than forty elements, including Xenon gas and Uranyl compounds. We will present the results of the structure solution from test models, the related methods and the instrumentation.

Keywords: Phasing, Heavy atoms, Softer X-rays

\section{MS1-P9 Automated system for data collection and data processing using microcrystals}

Keitaro Yamashita ${ }^{1}$, Kunio Hirata ${ }^{1}$, Yoshiaki Kawano ${ }^{1}$, Go Ueno $^{1}$, Kazuya Hasegawa ${ }^{2}$, Takashi Kumasaka ${ }^{1}$, Masaki Yamamoto $^{1}$

1. RIKEN SPring-8 Center, Advanced Photon Technology Division, Sayo, Japan

2. JASRI/SPring-8, Research \& Utilization Division, Sayo, Japan

email: k.yamashita@spring8.or.jp

On BL32XU, a micro-beam beamline at SPring-8, diffraction data are collected with typical horizontal beam size of $1 \mu \mathrm{m}$. This micro-beam has enabled data collection using small crystals, especially LCP crystals of membrane proteins that are important targets on the beamline. For crystals of $10 \mu \mathrm{m}$ or larger, a complete data-set could be obtained from a single crystal. However, for smaller crystals or weakly diffracting crystals, only small fraction of a complete data-set could be obtained from a single crystal, and therefore more crystals are required.

In order to facilitate crystal structure analysis using many microcrystals, we are developing an automated system for data collection and data processing from small crystals. Multiple data of small wedges can be collected using a single loop where many crystals are mounted. To find crystal positions accurately, raster diffraction scan using low-dose X-rays is performed in advance of data collection. We have developed a crystal alignment tool SHIKA based on the raster diffraction scan, which shows possible crystal position on 2D spot population map. SHIKA automatically processes new images in accordance with each raster diffraction scan. Found crystal positions are transferred to KUMA (a tool suggesting data collection strategy with predicted radiation damage) to start data collection immediately. Typical oscillation range of very small crystals is $10^{\circ}$ or $20^{\circ}$ for each crystal.

The datasets are processed automatically using XDS (Kabsch, 2010). Images that have few or no spots in low resolution area are discarded prior to data processing. After data processing, the datasets are hierarchically clustered based on unit cell dimensions using BLEND (Foadi, 2013). The clusters with large completeness are subjected to merging using XSCALE or Aimless (Evans, 2011). Outliers are detected based on pairwise correlation. This automated system has greatly reduced time and effort spent on beamline or at home.

Keywords: microbeam beamline, microcrystal, merging 